\title{
SOME NOTES ON FIRST STRONGLY GRADED RINGS
}

\author{
RASHID ABU-DAWWAS, ALA'A MESLEH, AND KHALDOUN AL-ZOUBI
}

Received 10 May, 2016

\begin{abstract}
Let $G$ be a group with identity $e$ and $R$ be an associative ring with a nonzero unity 1. Assume that $R$ is first strongly $G$-graded and $H=\operatorname{supp}(R, G)$. For $g \in H$, define $\alpha_{g}(x)=$ $\sum_{i=1}^{n_{g}} r_{g}^{(i)} x t_{g^{-1}}^{(i)}$ where $x \in C_{R}\left(R_{e}\right)=\left\{r \in R: r x=x r\right.$ for all $\left.x \in R_{e}\right\}, r_{g}^{(i)} \in R_{g}$ and $t_{g^{-1}}^{(i)} \in$ $R_{g-1}$ for all $i=1, \ldots \ldots, n_{g}$ for some positive integer $n_{g}$. In this article, we study $\alpha_{g}(x)$ and it's properties.
\end{abstract}

2010 Mathematics Subject Classification: 16W50; 13A02; 16D25; 46H10.

Keywords: Graded rings, first strongly graded rings

\section{INTRODUCTION}

Throughout this article, $R$ is an associative ring with nonzero unity 1 . For a ring $R$ and a subset $T$ of $R, C_{R}(T)=\{r \in R: r t=t r$ for all $t \in T\}$. For a group $G$, $Z(G)=\{g \in G: g x=x g$ for all $x \in G\}$. Let $G$ be a group with identity $e$. Then $R$ is said to be $G$-graded if there exist additive subgroups $R_{g}$ of $R$ such that $\mathbf{R}=$ $\bigoplus_{g \in G} \mathbf{R}_{g}$ where $R_{g} R_{h} \subseteq R_{g h}$ for all $g, h \in G$. For $x \in R, x=\sum_{g \in G} x_{g}$ where $x_{g}$ is the component of $x$ in $R_{g}$. Also, $\operatorname{supp}(R, G)=\left\{g \in G: R_{g} \neq 0\right\}$. Moreover, $R_{e}$ is a subring of $R$ and $1 \in R_{e}$. For more details, see [4]. Throughout this article, $H=\operatorname{supp}(R, G)$.

First strongly graded rings have been introduced by Refai in [5]. A $G$-graded ring $R$ is said to be first strongly graded if $1 \in R_{g} R_{g^{-1}}$ for all $g \in H$. $R$ is first strongly $G$-graded if and only if $H$ is a subgroup of $G$ and $\mathbf{R}_{g} \mathbf{R}_{h}=\mathbf{R}_{g h}$ for all $g, h \in H$. For more details, see [5].

Definition 1 ([4]). Let $R$ be a ring. Suppose that $\alpha: G \rightarrow A u t(R)$ and $\beta: G \times G \rightarrow$ $U(R)$ where $\operatorname{Aut}(R)$ is the group of automorphisms of $R$ and $U(R)$ is the group of units of $R$. In [4], $(R, G, \alpha, \beta)$ is said to be crossed system if the following conditions hold for all $g, h, s \in G$ and $a \in R$.

(1) $\alpha_{g}\left(\alpha_{h}(a)\right) \beta(g, h)=\beta(g, h) \alpha_{g h}(a)$. 
(2) $\beta(g, h) \beta(g h, s)=\alpha_{g}(\beta(h, s)) \beta(g, h s)$.

(3) $\beta(g, e)=\beta(e, g)=1$.

In [2], a $G$-graded ring $R$ is said to be crossed product over the support if $R_{g} \bigcap U(R) \neq \phi$ for all $g \in H$. In [1], it was shown that if $R$ is crossed product over the support, then $R$ is first strongly graded and then by [5], $H$ is a subgroup of $G$ with $\mathbf{R}_{g} \mathbf{R}_{h}=\mathbf{R}_{g h}$ for all $g, h \in H$.

Suppose that $R$ is crossed product over the support. We may choose the family $\left\{u_{g}\right\}_{g \in H}$ in $R$ such that $u_{g} \in R_{g} \bigcap U(R)$ for all $g \in H$ and assume that $u_{e}=1$. So, $R_{g}=R_{e} u_{g}=u_{g} R_{e}$ and $\left\{u_{g}\right\}_{g \in H}$ is a basis for the left (right) $R_{e}$-module $R$. Define the map $\alpha: H \rightarrow \operatorname{Aut}\left(R_{e}\right)$ by $\alpha(g)=\alpha_{g}$ where $\alpha_{g}(a)=u_{g} a u_{g}^{-1}$ for all $g \in H$ and $a \in R_{e}$. Also, define $\beta: H \times H \rightarrow U\left(R_{e}\right)$ by $\beta(g, h)=u_{g} u_{h} u_{g h}^{-1}$ for all $g, h \in H$. Then $\alpha$ and $\beta$ satisfy the conditions (1), (2) and (3) above (see [1]). Hence, $\left(R_{e}, H, \alpha, \beta\right)$ is a crossed system.

Assume that $R$ is first strongly $G$-graded. For $g \in H$, define $\alpha_{g}(x)=\sum_{i=1}^{n_{g}} r_{g}^{(i)} x t_{g^{-1}}^{(i)}$ where $x \in C_{R}\left(R_{e}\right), r_{g}^{(i)} \in R_{g}$ and $t_{g^{-1}}^{(i)} \in R_{g^{-1}}$ for all $i=1, \ldots \ldots, n_{g}$ for some positive integer $n_{g}$. In this article, we study $\alpha_{g}(x)$ and it's properties.

\section{Results}

In this section, we introduce our results.

Let $R$ be a $G$-graded ring and $X$ be a non-empty subset of $G$. Then $R_{X}=\bigoplus_{g \in X} R_{g}$. If $X$ is a subgroup of $G$, then $R_{X}$ is a subring of $R$. For more details, see [3]. We begin our results by the following.

Theorem 1. Consider the above crossed system $\left(R_{e}, H, \alpha, \beta\right)$. Suppose that $X$ is a subgroup of $H$ such that $X \subseteq Z(H) \cap \operatorname{Ker}(\alpha)$ and $\beta(x, y)=\beta(y, x)$ for all $(x, y) \in X \times X$. If $R_{e}$ is commutative, then $R_{X}$ is commutative.

Proof. Consider the family $\left\{u_{g}\right\}_{g \in H}$ above. Let $g, h \in X$ and $a_{g}, b_{h} \in R_{e}$. Then

$$
\begin{gathered}
\left(a_{g} u_{g}\right)\left(b_{h} u_{h}\right)=a_{g} \alpha_{g}\left(b_{h}\right) \beta(g, h) u_{g h}=a_{g} b_{h} \beta(g, h) u_{g h}= \\
b_{h} \alpha_{h}\left(a_{g}\right) \beta(h, g) u_{h g}=\left(b_{h} u_{h}\right)\left(a_{g} u_{g}\right) .
\end{gathered}
$$

Hence, $R_{X}$ is commutative.

Let $R$ be a first strongly $G$-graded ring (not necessary to be crossed product over the support). Then $R_{g} R_{g^{-1}}=R_{e}$ for all $g \in H$. So, for every $g \in H$, there exists $n_{g} \in \mathbf{Z}^{+}, r_{g}^{(i)} \in R_{g}$ and $t_{g^{-1}}^{(i)} \in R_{g^{-1}}$ such that $1=\sum_{i=1}^{n_{g}} r_{g}^{(i)} t_{g^{-1}}^{(i)}$ since $1 \in R_{e}$. 
Define $\alpha_{g}(x)=\sum_{i=1}^{n_{g}} r_{g}^{(i)} x t_{g^{-1}}^{(i)}$ for all $x \in C_{R}\left(R_{e}\right)$.

Theorem 2. Let $R$ be a first strongly graded ring. Then $\alpha_{g}$ is independent of the choice of $r_{g}^{(i)}$ 's and $t_{g-1}^{(i)}$ 's.

Proof. Let $n_{g}, n_{g}^{\prime} \in \mathbf{Z}^{+}$and $r_{g}^{(i)}, s_{g}^{(i)} \in R_{g}, t_{g^{-1}}^{(i)}, w_{g^{-1}}^{(i)} \in R_{g^{-1}}$ such that

$$
1=\sum_{i=1}^{n_{g}} r_{g}^{(i)} t_{g^{-1}}^{(i)}=\sum_{j=1}^{n_{g}^{\prime}} s_{g}^{(j)} w_{g^{-1}}^{(j)} \text {. }
$$

Let $x \in C_{R}\left(R_{e}\right)$. Then since $w_{g-1}^{(j)} r_{g}^{(i)} \in R_{e}$,

$$
\begin{gathered}
\sum_{i=1}^{n_{g}} r_{g}^{(i)} x t_{g^{-1}}^{(i)}-\sum_{j=1}^{n_{g}^{\prime}} s_{g}^{(j)} x w_{g^{-1}}^{(j)}=1 \cdot\left(\sum_{i=1}^{n_{g}} r_{g}^{(i)} x t_{g^{-1}}^{(i)}\right)-\left(\sum_{j=1}^{n_{g}^{\prime}} s_{g}^{(j)} x w_{g^{-1}}^{(j)}\right) \cdot 1= \\
\sum_{j=1}^{n_{g}^{\prime}} \sum_{i=1}^{n_{g}} s_{g}^{(j)} w_{g^{-1}}^{(j)} r_{g}^{(i)} x t_{g^{-1}}^{(i)}-\sum_{j=1}^{n_{g}^{\prime}} \sum_{i=1}^{n_{g}} s_{g}^{(j)} x w_{g^{-1}}^{(j)} r_{g}^{(i)} t_{g^{-1}}^{(i)}=0 .
\end{gathered}
$$

The next lemma is fundamental for our next results.

Lemma 1. Let $R$ be a first strongly graded ring. If $r \in R$ such that $r R_{h}=\{0\}$ for some $h \in H$, then $r=0$.

Proof. Suppose that $r \in R$ and $h \in H$ such that $r R_{h}=\{0\}$. Then $r=r .1 \in r R_{e}=$ $r R_{h} R_{h^{-1}}=\left(r R_{h}\right) R_{h^{-1}}=\{0\}$, i.e., $r=0$.

Theorem 3. Let $R$ be a first strongly graded ring and $g \in H$. Then $\alpha_{g}(x)$ is the only element of $R$ satisfies $\alpha_{g}(x) a_{g}=a_{g} x$ for all $a_{g} \in R_{g}$. Moreover, $\alpha_{g}(x) \in$ $C_{R}\left(R_{e}\right)$ and if $x \in Z\left(R_{e}\right)$, then $\alpha_{g}(x) \in Z\left(R_{e}\right)$.

Proof. Let $a_{g} \in R_{g}$. Then $t_{g^{-1}}^{(i)} a_{g} \in R_{g^{-1}} R_{g}=R_{e}$ and $t_{g-1}^{(i)} a_{g}$ commutes with $x \in C_{R}\left(R_{e}\right)$ for all $i=1, \ldots . ., n_{g}$. So,

$$
\begin{gathered}
\alpha_{g}(x) a_{g}=\sum_{i=1}^{n_{g}} r_{g}^{(i)} x t_{g^{-1}}^{(i)} a_{g} \\
=\sum_{i=1}^{n_{g}} r_{g}^{(i)} t_{g^{-1}}^{(i)} a_{g} x=a_{g}\left(\sum_{i=1}^{n_{g}} r_{g}^{(i)} t_{g^{-1}}^{(i)}\right) x \\
=a_{g} \cdot 1 . x=a_{g} x .
\end{gathered}
$$

Let $x \in C_{R}\left(R_{e}\right)$ and $y \in R$ such that $r_{g}^{(i)} x=y r_{g}^{(i)}$ for all $i=1, \ldots ., n_{g}$. Then 


$$
\alpha_{g}(x)=\sum_{i=1}^{n_{g}} r_{g}^{(i)} x t_{g^{-1}}^{(i)}=\sum_{i=1}^{n_{g}} y r_{g}^{(i)} t_{g^{-1}}^{(i)}=y\left(\sum_{i=1}^{n_{g}} r_{g}^{(i)} t_{g^{-1}}^{(i)}\right)=y .1=y,
$$

i.e., $\alpha_{g}(x)$ is the only element satisfies $\alpha_{g}(x) a_{g}=a_{g} x$ for all $a_{g} \in R_{g}$. Since $R$ is first strongly graded, if $x \in R_{e}$, then $\alpha_{g}(x) \in R_{e}$. In particular, if $x \in Z\left(R_{e}\right)$, then $\alpha_{g}(x) \in Z\left(R_{e}\right)$. So, for $x \in Z\left(R_{e}\right)$ and $s \in R_{e}$,

$$
s \alpha_{g}(x)=1 . s \alpha_{g}(x)=\sum_{i=1}^{n_{g}} \sum_{j=1}^{n_{g}^{\prime}} r_{g}^{\prime(j)} t_{g^{-1}}^{\prime(j)} s r_{g}^{(i)} x t_{g^{-1}}^{(i)} .
$$

Since $t_{g^{-1}}^{\prime(j)} s r_{g}^{(i)} \in R_{e}$

$$
\begin{gathered}
s \alpha_{g}(x)=\sum_{i=1}^{n_{g}} \sum_{j=1}^{n_{g}^{\prime}} r_{g}^{\prime(j)} x t_{g^{-1}}^{(j)} s r_{g}^{(i)} t_{g^{-1}}^{(i)}=\left(\sum_{j=1}^{n_{g}^{\prime}} r_{g}^{(j)} x t_{g^{-1}}^{(j)}\right) . s .\left(\sum_{i=1}^{n_{g}} r_{g}^{(i)} t_{g^{-1}}^{(i)}\right)= \\
\alpha_{g}(x) . s .1=\alpha_{g}(x) s .
\end{gathered}
$$

If $a_{g} \in R_{g}$ and $w \in R_{e}$, then $w a_{g} \in R_{e} R_{g}=R_{g}$ and then

$$
\begin{gathered}
\left(\alpha_{g}(x) w\right) a_{g}=\alpha_{g}(x)\left(w a_{g}\right)=\left(w a_{g}\right) x=w\left(a_{g} x\right)=w\left(\alpha_{g}(x) a_{g}\right) \\
=\left(w \alpha_{g}(x)\right) a_{g}
\end{gathered}
$$

Which implies that $\left(\alpha_{g}(x) w-w \alpha_{g}(x)\right) R_{g}=\{0\}$. By Lemma $1, \alpha_{g}(x) w=w \alpha_{g}(x)$ and hence $\alpha_{g}(x) \in C_{R}\left(R_{e}\right)$.

Theorem 4. Let $R$ be a first strongly graded ring. Then the following hold:

(1) $\alpha_{g} \circ \alpha_{h}=\alpha_{g h}$ for all $g, h \in H$.

(2) $\alpha_{g}^{-1}=\alpha_{g^{-1}}$ for all $g \in H$.

(3) $\alpha_{g}(x b)=\alpha_{g}(x) \alpha_{g}(b)$ for all $g \in H$ and $x, b \in C_{R}\left(R_{e}\right)$.

Proof. Since $1 \in R_{e}, x=1 . x=\alpha_{e}(x) .1=\alpha_{e}(x)$ for all $x \in C_{R}\left(R_{e}\right)$. Let $g, h \in$ $H, a_{g} \in R_{g}$ and $a_{h} \in R_{h}$. Then $a_{g} a_{h} \in R_{g} R_{h}=R_{g h}$ and then

$$
\begin{gathered}
\alpha_{g h}(x)\left(a_{g} a_{h}\right)=\left(a_{g} a_{h}\right) x=a_{g}\left(a_{h} x\right)=a_{g}\left(\alpha_{h}(x) a_{h}\right)=\left(a_{g} \alpha_{h}(x)\right) a_{h} \\
=\left(\alpha_{g}(x)\left(\alpha_{h}(x)\right) a_{g}\right) a_{h}=\alpha_{g}(x)\left(\alpha_{h}(x)\right)\left(a_{g} a_{h}\right)
\end{gathered}
$$

for all $x \in C_{R}\left(R_{e}\right)$ which implies that $\alpha_{g}\left(\alpha_{h}(x)\right)=\alpha_{g h}(x)$ by Lemma 1 as $a_{g} a_{h}$ generates the $R_{e}$-submodule $R_{g h}$.

Let $g \in H, s_{g^{-1}}^{(j)} \in R_{g^{-1}}$ and $w_{g}^{(j)} \in R, j=1, \ldots . ., n_{g^{-1}}$ such that $1=\sum_{j=1}^{n_{g^{-1}}} s_{g^{-1}}^{(j)} w_{g}^{(j)}$.

Then for every $x \in C_{R}\left(R_{e}\right)$, 


$$
\begin{gathered}
\alpha_{g^{-1}}\left(\alpha_{g}(x)\right)=\sum_{j=1}^{n_{g^{-1}}} s_{g^{-1}}^{(j)}\left(\sum_{i=1}^{n_{g}} r_{g}^{(i)} x t_{g^{-1}}^{(i)}\right) w_{g}^{(j)}= \\
\sum_{j=1}^{n_{g-1}} s_{g^{-1}}^{(j)}\left(\sum_{i=1}^{n_{g}} r_{g}^{(i)} x t_{g^{-1}}^{(i)} w_{g}^{(j)}\right)=\sum_{j=1}^{n_{g-1}} s_{g^{-1}}^{(j)}\left(\sum_{i=1}^{n_{g}} r_{g}^{(i)} t_{g^{-1}}^{(i)} w_{g}^{(j)} x\right)= \\
\sum_{j=1}^{n_{g^{-1}}} s_{g^{-1}}^{(j)}\left(\sum_{i=1}^{n_{g}} r_{g}^{(i)} t_{g^{-1}}^{(i)}\right) w_{g}^{(j)} x=\sum_{j=1}^{n_{g}-1} s_{g^{-1}}^{(j)} \cdot 1 . w_{g}^{(j)} x=\left(\sum_{j=1}^{n_{g-1}} s_{g^{-1}}^{(j)} w_{g}^{(j)}\right) x \\
=1 . x=x
\end{gathered}
$$

which implies that $\left(\alpha_{g}\right)^{-1}=\alpha_{g^{-1}}$. For $x, b \in C_{R}\left(R_{e}\right)$ and $a_{g} \in R_{g}$,

$$
\begin{gathered}
\alpha_{g}(x b) a_{g}=a_{g}(x b)=\left(a_{g} x\right) b=\left(\alpha_{g}(x) a_{g}\right) b=\alpha_{g}(x)\left(a_{g} b\right)= \\
\alpha_{g}(x)\left(\alpha_{g}(b) a_{g}\right)=\left(\alpha_{g}(x) \alpha_{g}(b)\right) a_{g} .
\end{gathered}
$$

By Lemma $1, \alpha_{g}(x b)=\alpha_{g}(x) \alpha_{g}(b)$.

Theorem 5. Let $R$ be a first strongly graded ring. If $x \in C_{R}\left(R_{e}\right)$ and $g \in H$, then $x R_{g}=R_{g} x$ ( $x$ centralizes $R_{g}$ ) if and only if $\alpha_{g}(x)=x$.

Proof. Suppose that $R_{g}$ is centralized by $x$. Then for every $a_{g} \in R_{g}$,

$$
\alpha_{g}(x) a_{g}=\left(\sum_{i=1}^{n_{g}} r_{g}^{(i)} x t_{g^{-1}}^{(i)}\right) a_{g}=\sum_{i=1}^{n_{g}} r_{g}^{(i)} x t_{g^{-1}}^{(i)} a_{g} .
$$

Since $t_{g^{-1}}^{(i)} r_{g}^{(i)} \in R_{e}$

$$
\alpha_{g}(x) a_{g}=\sum_{i=1}^{n_{g}} r_{g}^{(i)} t_{g^{-1}}^{(i)} a_{g} x=\left(\sum_{i=1}^{n_{g}} r_{g}^{(i)} t_{g^{-1}}^{(i)}\right) a_{g} x=1 . a_{g} x=a_{g} x=x a_{g}
$$

as $x$ centralizes $R_{g}$. By Lemma $1, \alpha_{g}(x)=x$. Conversely, for every $a_{g} \in R_{g}$, $a_{g} x=\alpha_{g}(x) a_{g}=x a_{g}$, i.e., $x$ centralizes $R_{g}$

Corollary 1. Let $R$ be a first strongly graded ring. Then $Z(R)=\left\{x \in C_{R}\left(R_{e}\right)\right.$ : $\alpha_{g}(x)=x$ for all $\left.g \in H\right\}$.

Proof. Since $R$ is first strongly graded,

$$
Z(R)=\bigcap_{g \in H} C_{R}\left(R_{g}\right)=\left\{x \in C_{R}\left(R_{e}\right): x \in C_{R}\left(R_{g}\right) \text { for all } g \in H\right\} \text {. Note that if }
$$

$g \notin H$, then $R_{g}=\{0\}$ and then $C_{R}\left(R_{g}\right)=R$. By Theorem 5, $x \in C_{R}\left(R_{g}\right)$ if and only if $\alpha_{g}(x)=x$ and hence $Z(R)=\left\{x \in C_{R}\left(R_{e}\right): \alpha_{g}(x)=x\right.$ for all $\left.g \in H\right\}$. 
Remark 1. Also, it is nice to see that If $R$ is a first strongly graded ring, then $C_{R}\left(R_{e}\right)=$

$$
\begin{gathered}
\left\{x=\sum_{g \in H} x_{g} \in R: x_{g} \in R_{g} \text { with } c x_{g}=x_{g} c \text { for all } g \in H \text { and for all } c \in R_{e}\right\} \\
=\left\{x=\sum_{g \in H} x_{g} \in R: x_{g} \in\left(R_{g} \bigcap C_{R}\left(R_{e}\right) \text { for all } g \in H\right\}\right. \\
=\bigoplus_{g \in H}\left(R_{g} \bigcap C_{R}\left(R_{e}\right)\right) .
\end{gathered}
$$

Note that for $g \notin H, R_{g}=\{0\}$ and then $x_{g}=0$.

The next result is a generalization of Corollary 1.

Theorem 6. Let $R$ be a first strongly graded ring and $X$ be a subgroup of $H$. Then $C_{R}\left(R_{X}\right)=$

$$
\begin{gathered}
\left\{t=\sum_{g \in H} t_{g} \in R: t_{g} \in C_{R}\left(R_{e}\right) \bigcap R_{g}, \alpha_{x}\left(t_{g}\right)=t_{x g x^{-1}} \text { for all } g \in H \text { and for all } x \in X\right. \\
=\left\{t \in C_{R}\left(R_{e}\right): \alpha_{x}(t)=t \text { for all } x \in X\right\} .
\end{gathered}
$$

Proof. Let $t=\sum_{g \in H} t_{g} \in C_{R}\left(R_{X}\right)$ where $t_{g} \in R_{g}$. Since $R_{e} \subseteq R_{X}, t \in C_{R}\left(R_{e}\right)$ and then by Remark $1, t_{g} \in C_{R}\left(R_{e}\right)$ for all $g \in H$. Let $x \in X$. Then for every $s_{x} \in R_{x}$,

$$
s_{x} \sum_{g \in H} t_{g}=\sum_{g \in H} t_{g} s_{x}
$$

since $t \in C_{R}\left(R_{X}\right)$. As $t_{g} \in C_{R}\left(R_{e}\right)$, by Theorem 3,

$$
\sum_{g \in H} \alpha_{x}\left(t_{g}\right) s_{x}=\sum_{g \in H} t_{g} s_{x}
$$

Since $R_{x} R_{g} R_{x^{-1}} R_{x}=R_{x g}$, for all $g \in H$ and for all $x \in X$,

$$
\alpha_{x}\left(t_{g}\right) s_{x}=t_{x g x^{-1} s_{x}} \text { for all } s_{x} \in R_{x} .
$$

Choose $a_{x}^{(i)} \in R_{x}$ and $b_{x^{-1}}^{(i)} \in R_{x^{-1}}$ where $i=1, \ldots . ., n_{x}$ for some positive integer $n_{x}$ such that

$$
1=\sum_{i=1}^{n_{x}} a_{x}^{(i)} b_{x^{-1}}^{(i)}
$$

Then 


$$
\begin{gathered}
\alpha_{x}\left(t_{g}\right)=\alpha_{x}\left(t_{g}\right) .1=\alpha_{x}\left(t_{g}\right) \sum_{i=1}^{n_{x}} a_{x}^{(i)} b_{x^{-1}}^{(i)}=\sum_{i=1}^{n_{x}} \alpha_{x}\left(t_{g}\right) a_{x}^{(i)} b_{x^{-1}}^{(i)}= \\
\sum_{i=1}^{n_{x}} t_{x g x^{-1}} a_{x}^{(i)} b_{x^{-1}}^{(i)}=t_{x g x^{-1}} \sum_{i=1}^{n_{x}} a_{x}^{(i)} b_{x^{-1}}^{(i)}=t_{x g x^{-1}} .
\end{gathered}
$$

For the converse, Suppose that $t=\sum_{g \in H} t_{g} \in R$ where $t_{g} \in C_{R}\left(R_{e}\right) \bigcap R_{g}$ and $\alpha_{x}\left(t_{g}\right)=$ $t_{x g x^{-1}}$ for all $g \in H$ and for all $x \in X$. Then for every $s_{x} \in R_{x}$,

$$
s_{x} t=\sum_{g \in H} s_{x} t_{g}=\sum_{g \in H} \alpha_{x}\left(t_{g}\right) s_{x}=\sum_{g \in H} t_{x g x^{-1}} s_{x}=\sum_{r \in H} t_{r} s_{x}=t s_{x}
$$

which implies that $t \in C_{R}\left(R_{X}\right)$.

Theorem 7. Let $R$ be a first strongly graded ring such that $R_{e}$ is commutative. If $X$ is a subgroup of $H$ such that $X \subseteq Z(G)$ and $\alpha_{g}(a)=$ a for all $g \in H$ and $a \in R_{e}$, then $J \cap C_{R}\left(R_{X}\right) \neq\{0\}$ for every non-zero two sided ideal $J$ of $R$.

Proof. Let $J$ be a nonzero two sided ideal of $R$. Let $x \in X$ and $s_{x} \in R_{x}$. Define $K_{S_{x}}: R \rightarrow R$ by

$$
K_{s_{x}}(t)=K_{s_{x}}\left(\sum_{g \in H} t_{g}\right)=s_{x} \sum_{g \in H} t_{g}-\sum_{g \in H} t_{g} s_{x}=\sum_{j \in H} k_{j} .
$$

Note that $k_{x}=s_{x} t_{e}-t_{e} s_{x}=\alpha_{x}\left(t_{e}\right) s_{x}-t_{e} s_{x}=t_{e} s_{x}-t_{e} s_{x}=0$. On the other hand, $k_{x g}=s_{x} t_{g}-t_{g} s_{x} \in R_{g x}=R_{x g}$ might be zero or nonzero. Thus the number of elements in $\operatorname{supp}\left(K_{s_{x}}(t)\right.$ is less than $\operatorname{supp}(t)$. Moreover,

$$
C_{R}\left(R_{X}\right)=\bigcap_{x \in X, s_{x} \in R_{x}} \operatorname{Ker}\left(K_{s_{x}}\right)
$$

Let $t=\sum_{g \in H} t_{g} \in J$ be a nonzero element. We may assume that $t_{e} \neq 0$. Otherwise, there exists a nonzero $t^{\prime}=\sum_{g \in H} t_{g}^{\prime} \in J$ such that $t_{e}^{\prime} \neq 0$. So, there exists $y \in G$ such that $t_{y} \neq 0$. Also, there exists $b_{y^{-1}}^{(i)} \in R_{y^{-1}}$ such that $b_{y^{-1}}^{(i)} t_{y} \neq 0$ where $i=1, \ldots . ., n_{y}$ for some positive integer $n_{y}$, this is because if $b_{y^{-1}}^{(i)} t_{y}=0$ for all $i=1, \ldots . ., n_{y}$, then we choose $a_{y}^{(i)} \in R_{y}$ such that

$$
\sum_{i=1}^{n_{y}} a_{y}^{(i)} b_{y^{-1}}^{(i)}=1
$$


and then

$$
t_{y}=1 . t_{y}=\sum_{i=1}^{n_{y}} a_{y}^{(i)} b_{y^{-1}}^{(i)} t_{y}=0 .
$$

Hence, for every $t \in J$ there exists $b_{y^{-1}}^{(j)} t=t^{\prime}=\sum_{g \in H} t_{g}^{\prime}$ in $J$ such that $t_{e}^{\prime}=b_{y^{-1}}^{(j)} t_{y} \neq$ 0 and $|\operatorname{supp}(t)| \geq\left|\operatorname{supp}\left(t^{\prime}\right)\right| \geq 1$. Now, we assumed that $t=\sum_{g \in H} t_{g} \in J$ such that $t_{e} \neq 0$. If $t \in C_{R}\left(R_{X}\right)$, then it is done. Suppose that $t \notin C_{R}\left(R_{X}\right)$. Then there exists $x \in X$ and $s_{x} \in R_{x}$ such that $K_{s_{X}}(t) \neq 0$. Since $K_{s_{x}}(t) \in J$, we find an element in $J$ with smaller support. Keep on this procedure, we will stop since $\operatorname{supp}(t)$ is finite. Thus, we will find an element $\xi=\sum_{g \in H} \xi_{g} \in J \bigcap C_{R}\left(R_{X}\right)$ such that $\xi_{e} \neq 0$.

\section{ACKNOWLEDGEMENT}

The authors give great thanks to the referee who contributed to the wonderful output of the article.

\section{REFERENCES}

[1] R. Abu-Dawwas, "More on crossed product over the support of graded rings," International Mathematical Forum, vol. 5, no. 63, pp. 3121-3126, 2010.

[2] R. Abu-Dawwas and M. Refai, "Further results on graded prime submodules," International Journal of Algebra, vol. 4, no. 28, pp. 1413-1419, 2010.

[3] G. Karpilovsky, The algebraic structure of crossed products. North Holland, Amsterdam: North Holland Mathematics Studies, 118, 1987.

[4] C. Nastasescu and F. Oystaeyen, Methods of graded rings. Berlin: Springer, Lecture Notes in Mathematics, 2004, vol. 1836.

[5] M. Refai, "Various types of strongly graded rings." Abhath Al-Yarmouk Journal (Pure Sciences and Engineering Series)., vol. 4, no. 2, pp. 9-19, 1995.

Authors' addresses

\section{Rashid Abu-Dawwas}

Yarmouk University, Department of Mathematics, 21163 Irbid, Jordan

E-mail address: rrashideyu.edu.jo

\section{Ala'a Mesleh}

Yarmouk University, Department of Mathematics, 21163 Irbid, Jordan

E-mail address: alaa.mesleh9gegmail.com

\section{Khaldoun Al-Zoubi}

Jordan University of Science and Technology, Department of Mathematics and Statistics, 22110 Ramtha, Jordan

E-mail address: kfzoubi@ just. edu. jo 